\title{
Respiratory infections in the first year of life in children at risk of developing atopy
}

\author{
J J COGSWELL，D F HALLIDAY，J R ALEXANDER
}

\begin{abstract}
Ninety-two infants, each of whom had one parent with asthma or hay fever, were followed up from birth to the age of 1 year and 72 to the age of three years. During the first year of life respiratory symptoms, eczema, and respiratory viral infections were all reported. Within the first year 24 babies developed eczema; 28 had a wheal of $1 \mathrm{~mm}$ in diameter or more on prick skin testing with cutaneous allergens. Forty-three children had one or both of these characteristics and formed an atopic subgroup; by the same criteria, 49 children were non-atopic.

The number of respiratory infections in the two groups was not significantly different; similar viruses were isolated from both groups. These viruses were associated with both upper and lower respiratory tract infections. Wheezing was a clinical feature in 12 children during lower respiratory tract infections. Of these babies six were atopic in the first year of life. Of the six non-atopic babies, one had eczema in the second year and five children developed raised total serum IgE values within the 3 years.
\end{abstract}

\section{Introduction}

Respiratory infection is very common in young children. When it is accompanied by wheezing a diagnosis of wheezy bronchitis is frequently made. Respiratory viruses are known to be strongly associated with wheezing at this age. ${ }^{12}$

The relationship between virus-associated wheezing in early life and the later development of asthma remains unclear. Many children under school-age with wheezy bronchitis outgrow their symptoms, while others develop frank asthma in later years. Some regard wheezy bronchitis and asthma as extremes of a single disorder, ${ }^{3}$ and family studies suggest that both wheezy conditions share a common genetic basis. ${ }^{4}$

In this study we examined prospectively the atopic status of babies who wheezed in association with respiratory tract infections in the first year of life. A cohort of infants at risk of developing atopic disease was identified at birth and followed up regarding respiratory illness, infective agents, and atopic characteristics.

\section{Patients and methods}

Study design-The babies were selected for study before birth on the grounds that one parent gave a history of hay fever or asthma. This selection was designed to provide a cohort of children in whom about

Department of Paediatrics, Poole General Hospital, Poole, Dorset BH15 2JB

J J COGSWELL, MD, FRCP, consultant paediatrician

Virology Unit, Public Health Laboratory, Poole General Hospital, Poole, Dorset BH15 2JB

D F HALLIDAY, FIMLS, chief medical laboratory scientific officer

School of Mathematics, Statistics, and Computing, Thames Polytechnic, Woolwich, London SE18 GPF

J R ALEXANDER, BSC, MSC, medical statistician half would be expected to develop evidence of atopy; those without atopic signs acted as controls. Since this study was not designed to investigate the genetic basis of wheezy bronchitis or asthma, skin testing of the parents was not carried out. Consent from the pregnant mother was obtained after full explanation that the baby would be subjected to venepunctures and the passage of a soft nasal catheter during the course of the study. Parents could remove their babies from the study at any time. All the babies were born in the maternity department of a district general hospital. The mean birth weight was $3.28 \mathrm{~kg}$ and 79 infants were breast fed. The 92 families of the babies at 1 year old fell into the following social classes: class I, $10 \%$; class II, $36 \%$; class III, $43 \%$; class IV, $7 \%$; class V, $0 \%$; and others, $3 \%$.

Clinical assessment-All babies were examined at birth, 3 months, 6 months, and 12 months. At each hospital visit a clinical and environmental history was taken and added to by a daily record of respiratory symptoms and eczema kept by the parents. Clinical examination of the skin and respiratory tract was carried out. A diagnosis of eczema was made when the skin of the face or flexures showed roughening, redness, or intense pruritus. Transient spots or cradle caps were not regarded as eczema. At each hospital visit skin sensitivity testing was carried out by a prick test using eight common allergens: Dermatophagoides pteronyssimus, cows' milk, cat fur, egg, mixed grass pollen, dog hair, and budgerigar and mixed threshings. These allergens were chosen to represent those to which a baby under 1 year might be exposed. The choice was arbitrary, but the number of allergens was restricted by the technical and ethical considerations of multiple prick tests in very young children. A control solution and a $1 / 1000$ histamine solution (Bencard) were also used. The diameter of the wheal was recorded at 15 minutes and a wheal of $\geqslant 1 \mathrm{~mm}$ in diameter was regarded as positive.

Laboratory investigations-Whenever possible a venepuncture was performed on each child at every visit. When sufficient blood was obtained it was examined for full blood count, virus antibody titres, and immunoglobulins. The serum sent to the virus laboratory was examined by complement-fixation tests with the following viral antigens: Influenza virus $A, B$, and $C$, parainfluenza virus type $I$, adenovirus, mumps virus $S$ and $V$, respiratory syncytial virus, herpes simplex virus, measles virus, Mycoplasma pneumoniae, and psittacosis. A four-fold increase in antibody titre was regarded as serological evidence of a preceding viral infection. The serum was stored at $-20^{\circ} \mathrm{C}$ until analysed. Serum IgG, IgM, and IgA concentrations were measured by a quantitative single radial diffusion method. Total serum IgE was measured by a direct radioimmunoassay technique using paper discs as solid phase Paper Radio Immunosorbent Test (PRIST) (Phadebas). ${ }^{5}$ Whenever a baby developed respiratory symptoms the parents were asked to telephone a member of the research team within 24 hours. The family was then visited at home and the clinical condition of the baby recorded. A clinical diagnosis was made (for example, cold, croup, or bronchitis) according to criteria used in the 1965 Medical Research Council survey. ${ }^{6}$ Nasopharyngeal secretions were taken from the baby by suction using a fine catheter passed intranasally and collected in a mucus trap. ${ }^{7}$ The pharyngeal aspirate was transported without delay in a vacuum flask at $0^{\circ} \mathrm{C}$ to the virus laboratory. The secretions were washed out into virus transport media and any mucus homogenised by passing through a hypodermic needle from a syringe under pressure. The suspension obtained was inoculated into monolayer tissue cultures of Rhesus monkey kidney, HEP-2, and MRC-5 cells. After incubation isolates were identified by appropriate haemadsorption inhibition, neutralisation, or indirect fluorescent antibody techniques. To serve as controls 75 pharyngeal aspirates were obtained from the same babies at a time when they had no respiratory symptoms either before the pharyngeal aspirate was taken or for a week afterwards.

Statistical analysis-This was carried out with a computer package. Except where otherwise stated in the results, means were compared using Student's $t$ test or the analysis of variance, and contingency tables were analysed by means of the standard $\chi^{2}$ test. As is customary practice, logarithms of immunoglobulin values were analysed where 
appropriate. In our presentation of the IgE results we have used the median rather than the mean because of the very skew nature of the distribution.

\section{Results}

In the first year of life a mean of $3 \cdot 1$ respiratory infections occurred among the 92 babies. Fifteen episodes of wheeze with lower respiratory tract infections were recorded in 12 children. The remaining 269 infections were of the upper respiratory tract. There were no cases of bronchiolitis and no baby required hospital admission under 1 year of age.

Forty-three infants were defined as atopic by the presence of eczema or at least one positive skin test (wheal $\geqslant 1 \mathrm{~mm}$ in diameter) or both in the first year of life. Although total serum IgE values were not used in our definition, they were significantly higher in the atopic group at 3 months $(p<0.05), 6$ months $(p<0.01), 24$ months $(p<0.05)$, and 36 months of age $(p<0.01)$ (see figure). The atopic babies had a mean number of respiratory infections of 3.0 compared with 3.6 for the 49 non-atopic children. The difference was not significant $(\mathrm{p}>0.50)$.

Similar respiratory viruses were isolated from both the atopic and non-atopic groups (see table). The isolation rates for the symptomatic children and 75 asymptomatic controls were $19 \%$ and $4 \%$ respectively. A further 18 respiratory viruses were identified serologically by a fourfold rise in antibody titre in the first year (see table). Respiratory viruses were isolated during five of the 15 lower respiratory tract infections with wheezing: respiratory syncytial virus (two), parainfluenza virus 3 (two), and rhinovirus type $M$ (one). These same

Number of viruses isolated in the first year of life in atopic and non-atopic babies

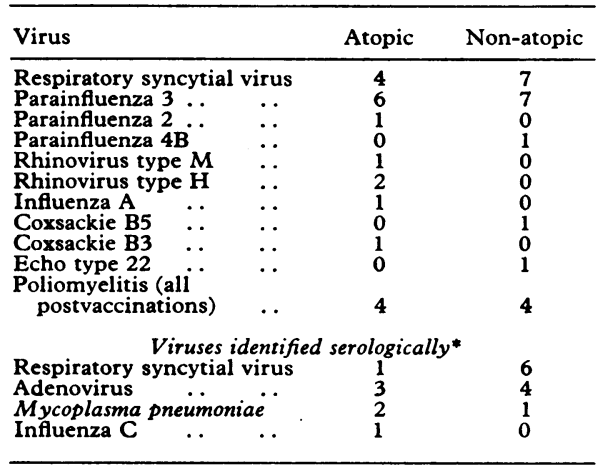

* By fourfold rise in complement-fixing antibody

viruses were also isolated from babies with only upper respiratory symptoms.

Both the atopic and non-atopic babies had upper and lower respiratory tract infections. In the non-atopic babies (49) there were 149 upper respiratory infections and six episodes of lower respiratory tract infection with wheeze in six babies. In the atopic group (43), six babies had a total of nine lower respiratory tract infections with wheezing, and 120 upper respiratory infections were recorded.

Seventy-two of the children were followed up for a further two years. The allergic status of the six wheezy children classified as nonatopic in the first year of life changed; one of the six developed eczema at over 1 year old. Moreover, the total serum IgE values in these six children remained consistently high; of 25 measurements on the six children over three years, $80 \%$ were above the median for the group as a whole (see figure). The mean IgA concentration on the sera of both groups of babies at 3 months of age was $0.16 \mathrm{~g} / \mathrm{l}$.

\section{Discussion}

Many studies have shown that respiratory illness during the first year of life is frequently due to viral infections. This present study supports the evidence that the same viruses cause both upper and lower respiratory tract infections and whether a baby develops a wheeze or not is not determined by the infecting agent.

In this longitudinal study we examined the relationship of atopy to the number and clinical outcome of respiratory infections within the first year of life. Those babies with clinical signs of atopy before their first birthday had not suffered more respiratory infections within the same year than the non-atopic babies. Although asthmatic children have a greater frequency of viral infections, ${ }^{8}$ our findings do not support this view.

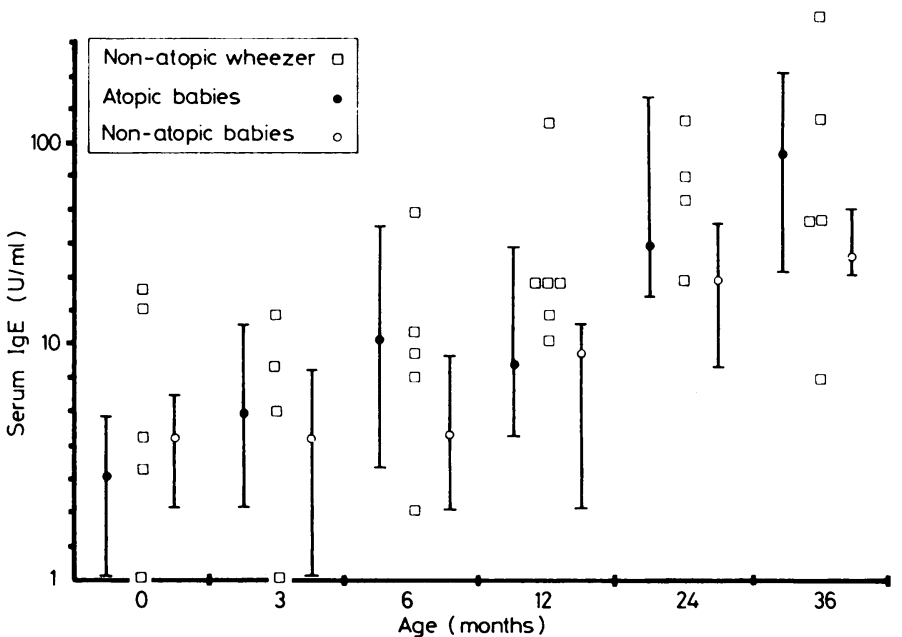

Serum IgE concentrations in atopic and non-atopic babies showing median with upper and lower quartiles. (The 29 measurements in the six wheezy children who were non-atopic in the first year are also shown.)

As many children were classed as non-atopic with wheeze and lower respiratory infection in the first year of life as overtly atopic children. More recurrent wheezy episodes occurred, however, in the atopic group in their first year. When the six wheezy babies, who were classed as non-atopic in the first year, were followed-up for a further two years, atopic characteristics in the form of eczema or raised total serum IgE concentrations tended to develop.

Transient IgA deficiency at 3 months of age predisposes to the development of eczema and positive skin tests. ${ }^{910}$ In this study we were unable to show any difference in serum IgA values at 3 months of age between our atopic and non-atopic children; all the results were within the expected normal range. Difference in study design, definition criteria, and percentage of cohort available for follow up may have influenced our findings when compared with those of others.

Studies of the genetic basis of asthma and wheezy bronchitis suggest that these wheezy illnesses share a common genetic defect. ${ }^{4}$ In this prospective study of a group of children at risk for atopic disorders we found that the atopic constitution is an important factor in determining whether a baby will respond to a respiratory virus infection by wheezing. Evidence of the atopic state may be apparent at the time of the first wheezy episode or may only become apparent after further follow up. Prediction of the atopic constitution was not improved by serum IgA estimations at 3 months.

We gratefully acknowledge the technical and nursing assistance of Marie Dawson, Christine Newsome, Ann Palmer, and Joan Wignall. We thank $\mathrm{Dr} W \mathrm{~L}$ Hooper for advice and encouragement. Financial support for this work was received from the Wessex Regional Health Authority, the Asthma Research Council, and the Medical Research Council.

Correspondence should be addressed to Dr J J Cogswell, Paediatric Department, Poole General Hospital, Longfleet Road, Poole, Dorset BH15 2JB. 


\section{References}

${ }^{1}$ Horn MEC, Brain E, Gregg I, Yealland SK, Inglis JM. Respiratory viral infection in childhood. A survey in general practice, Roehampton 1967-72. 7 Hyg 1975;74:157-68.

2 Mitchell I, Inglis H, Simpson H. Viral infections in wheezy bronchitis and asthma in children. Arch Dis Child 1976;51:707-11.

3 Williams H, McNicol KN. Prevalence, natural history and relationship of wheezy bronchitis and asthma in children. An epidemiological study. Br Med ₹ 1969 ;iv:321-5.

4 Sibbald B, Horn MEC, Gregg I. A family study of the genetic basis of asthma and wheezy bronchitis. Arch Dis Child 1980;55:354-57.

5 Johansonn SGO, Berglund A, Kjellman NIM. Comparison of IgE values as determined by different solid phase radioimmunoassay methods. Clin Allergy $1976 ; 6: 91-8$.
- Gardner PS. Virus infections and respiratory disease of childhood. Review article. Arch Dis Child $1968 ; 43: 629-44$

${ }^{7}$ McQuillin J, Gardner PS. Rapid diagnosis of respiratory syncytial virus infection by immunofluorescent antibody technique. $\mathrm{Br} \mathrm{Med} \mathcal{F} 1968$ : $602-5$.

${ }^{8}$ Minor TE, Baker JW, Dick EC, et al. Greater frequency of viral infections in asthmatic children as compared with their non-asthmatic siblings. $f$ Pediatr $1974 ; 85: 472-7$.

- Taylor B, Norman AP, Orgel HA, Stokes CR, Turner MW, Soothill JF. Transient IgA deficiency and pathogenesis of infantile atopy. Lancet 1973 ;ii:111-3.

${ }^{10}$ Soothill JF, Stokes CR, Turner MW, Norman AP, Taylor B. Predisposing factors and the development of reaginic allergy in infancy. Clin Allergy $1976 ; 6: 305-19$.

(Accepted 28 fanuary 1982)

\section{SHORT REPORTS}

\section{Incidence of pregnancy after laparoscopy and hydrotubation}

Many doctors experienced in managing infertility have observed that pregnancy may ensue soon after tests of tubal patency. Rubin ${ }^{1}$ reported an $18 \%$ incidence of pregnancy after gas insufflation, while hysterosalpingography has been credited with incidences of $25-40 \%{ }^{2}$ Laparoscopy and dye hydrotubation may also have a therapeutic effect in addition to being a diagnostic procedure. We conducted a retrospective study of conceptions that occurred before and after laparoscopy based on life-table analysis.

\section{Patients, methods, and results}

A total of 172 women (median age 27 years) presented to one infertility clinic over two years; $101(59 \%)$ presented with primary infertility and 71 $(41 \%)$ with secondary infertility. All had been trying to conceive for more than one year (median two years)

At the first visit a full history was obtained and clinical examination carried out on both partners; a postcoital test was then done at mid cycle. Men with obvious semen abnormalities, women with obvious menstrual or endocrine abnormalities, and spontaneous defectors were excluded from the study. The remainder were put on a waiting list for laparoscopy and followed up for spontaneous conception in the waiting period (median duration seven months). Patients with obvious tubal abnormalities on laparoscopy were excluded. The remainder were followed up for one to 24 months, which terminated in either pregnancy or further investigations. The time available for conception both before and after laparoscopy thus varied between patients.

If hydrotubation did not affect the probability of conception some of the subfertile women presenting to a clinic would conceive spontaneously and the remainder, who would undergo laparoscopy, would therefore form a group with relatively lower fertility. If a greater incidence of conception was found in the group who underwent laparoscopy this would suggest that hydrotubation promoted fertility.

Each month after presentation or laparoscopy the number of women who had conceived and the number who had withdrawn from follow-up were noted. The number who were available for conception each month and the number of expected pregnancies if the risks of pregnancy were equal in the two groups were calculated. The expected number of pregnancies was

Details of patients excluded from study, and those followed up before and after laparoscopy

\begin{tabular}{lcc}
\hline & $\begin{array}{c}\text { Before } \\
(\mathrm{n}=172)\end{array}$ & $\begin{array}{c}\text { After } \\
(\mathrm{n}=65 ; 63+2 *)\end{array}$ \\
\hline $\begin{array}{l}\text { Patients excluded: } \\
\text { Laparoscopy elsewhere }\end{array}$ & $2{ }^{*}$ & \\
Spontaneous defectors & $35 \%)$ & $9(13 \%)$ \\
Immediate surgery/hormone treatment & 3 & \\
Already pregnant at postcoital test & 6 & \\
Partner had problem & 42 & 3 \\
Others & 81 & 38 \\
Total No for follow-up & $17(21 \%)$ & $25(66 \%)$ \\
Spontaneous conceptions & 1 & 10 \\
Delayed surgery/hormone treatment & 63 & 3 \\
Other & & \\
Laparoscopy & & \\
\hline
\end{tabular}

compared with the observed number using life-table analysis with the standard method of Mantel ${ }^{3}$ We followed Haybittle and Freedman ${ }^{4}$ in omitting the continuity correction, as the number of months for which the women were at risk varied considerably.

Of the 172 women, 81 were available for follow-up in the group awaiting laparoscopy. Sixty-five underwent laparoscopy, of whom 38 were available for follow-up (table). The incidence of conception in the two groups was compared using a single-sided normal deviate test; this gave $Z=4 \cdot 77$, $p<0.001$. Thus it was unlikely that the proportion conceiving after laparoscopy was less than or equal to that conceiving before laparoscopy. This was not, however, a reliable comparison as the number of months for which the women were at risk varied considerably. Life-table analysis, which takes into account the number of months at risk, showed that although the observed number of conceptions after laparoscopy was 25 , the expected number was 13.27 , and that variance was 8.34 . The standard normal deviate was $4 \cdot 07$. The probability of a value as high as this (one sided) is $<0.001$. The apparent increase in fertility after laparoscopy was therefore unlikely to be due to chance. By comparing the number of non-attenders in the two groups $\left(\chi^{2}=1.64, \mathrm{df}=1, \mathrm{p}>0 \cdot 10\right)$, it seemed unlikely that the apparent increase in fertility after laparoscopy could be explained on the basis of an excess of unreported pregnancies in the women awaiting laparoscopy.

\section{Comment}

In the absence of any obvious abnormality spontaneous conception in an infertile woman is difficult to explain. Leeton and Selwood found that in patients undergoing laparoscopy those with multiple tubal tortuosities had a higher incidence of conception and attributed this to minimal tubal obstruction, which could be alleviated by tubal insufflation. We think the reason for a higher incidence of conception after laparoscopy is due to removal of minor obstruction such as fine intraliminal adhesions or inspissated mucous plugs.

We thank Dr Richard Kerr-Wilson, Mrs Monica Green, and Mrs Joan Searle for their help in collecting the relevant data for this study.

${ }^{1}$ Rubin I. Utero-tubal insufflation. St Louis: C V Mosby, 1947.

2 Wahby O, Subrero A, Epstein J. Hysterosalpingography in relation to pregnancy and its outcome in infertile women. Fertil Steril 1966;17: 520-30.

${ }^{3}$ Mantel N. Evaluation of survival data and two new rank order statistics arising in its consideration. Cancer Chemotherapy Reports 1966;50: 163-70.

4 Haybittle J, Freedman L. Some comments on logrank test statistic in clinical trial application. Statistician $1979 ; 28: 199-208$.

${ }^{5}$ Leeton J, Selwood T. The tortuous tube: pregnancy rates following laparoscopy and hydrotubation. Aust NZ $\mathcal{F}$ Obstet Gynaecol 1978;18: 259-62.

(Accepted 28 December 1981)

Department of Obstetrics and Gynaecology, St Helier Hospital, Surrey, and Department of Clinical Epidemiology and Social Medicine, St George's Hospital, London SW17 ORE

PARAMJIT LUTHRA, MRCOG, obstetric registrar

J MARTIN BLAND, PHD, MSC, lecturer in medical statistics

STUART L STANTON, FRCS, MRCOG, consultant obstetrician and gynaecologist (St Helier Hospital), and honorary senior lecturer 